\title{
Failure Modes of Deep Foundation Pit Supporting
}

\author{
Guo $\mathrm{Li}^{1, \mathrm{a}}$, He Peng-li ${ }^{2, \mathrm{~b}}$ \\ ${ }^{1}$ Luoyang Institute of Science and Technology, Luoyang, Henan, 471023, China \\ ${ }^{2}$ Luoyang Institute of Science and Technology, Luoyang, Henan, 471023, China \\ agldrmj@126.com, bhpl7609@126.com
}

Keywords: deep foundation pit; failure mode; instability; reliability

\begin{abstract}
In order to overcome the defects of the safety factor of foundation pit stability, reliability theory has been applied to the stability analysis of foundation pit. The theory depends on the establishment of correct failure mode and function of foundation pit. Through the analysis of the influence factors of the stability of deep foundation pit, the failure modes of deep foundation pit supporting were scientifically summarized from failure mechanism. Several failure modes are given, and they are coupled with each other and have a certain degree of correlation. Thus, it laid the foundation for the calculation of the foundation pit support system reliability.
\end{abstract}

\section{Introduction}

As is known to all, the deep foundation pit support is a very complex temporary systematic engineering. The reason of the complexity lies in the structural characteristic, regionality and variability of the underground structure and rock and soil materials, and the safety reserve of the supporting system is relatively low [1]. In order to realize the optimization of support, the stability of the deep foundation pit supporting should be guaranteed in the first place [2].

For the control of the stability, the most promising design method is based on the reliability theory of the limit state. In this method, the failure mode of foundation pit support is sought, and the limit state equation is established, and the reliability index is obtained by solving the equation. With the aid of this, the stability of the supporting system is evaluated [3]. And to obtain the reliability index, the failure mode of the foundation pit supporting system should be established [4]. By means of analysis of the influence factors and failure mechanism of the stability of support system, the failure mode of foundation pit is established. Thus, the more accurate help will be provided to the reliability of the calculation.

\section{Influence factors of support failure}

Through theoretical analysis and a large number of practical verification, the factors that affect the stability of the supporting system can be attributed to external factors and internal factors [5]. Internal factors mainly include parameters variability of rock and soil, discontinuity of rock and soil material, nonlinear of underground structure and rock and soil materials, the interaction between rock and soil mass and supporting structure, the space-time effect of foundation pit. External factors mainly include accidental factors, construction process, construction management, inappropriate design, ineffective control of groundwater, the lack of informationization construction [6].

Internal Factors Related to the Rock and Soil Mass. The influence of internal factors on the stability of the support system is mainly due to the variability of the rock and soil materials, and this variability is mainly reflected in the structural and random characteristic [7]. So it has brought a lot of trouble to solving the earth pressure. Whether Coulomb earth pressure model, or Rankine earth pressure model, or modified earth pressure model, the soil parameters c and $\varphi$ must be determined [8].

Through statistical analysis, it was observed that these two parameters are the main indicators which affect the earth pressure, and they have certain pertinence. After excavation, the foundation pit often shows a certain space-time effect. The passive earth pressure and horizontal displacement of retaining structure near the corner of the foundation pit is smaller, then gradually increase, and reach 
the maximum in the middle of the foundation pit. And the law of active earth pressure is opposite. These are main manifestations of space-time effect.

Many of the supporting systems are now made of the space truss that are connected. Regardless of the material of the truss, after the construction, the truss has some degree of shrinkage and creep, especially the concrete member. This creep can cause the stress relaxation of the bar, so that the stress level of the member should be reduced. At the same time, the supporting rod is directly or indirectly contact with the soil, so the joint strength design and construction design are required in connection. For example, the anchor point, the links between the rods are also treated against water and rust. The friction between the supporting structure and the soil should be considered in the support design in order to reduce calculation value of the active earth pressure. However, this problem is seldom considered in the calculation of earth pressure. And these factors are interactional and coupled, they shall be integrated and treated. Many foundation pit designers do not pay attention to the great harm of this kind of problem. This could be seen from: when the strength index is selected, the test method is inappropriate, model is unreasonable, calculation method is lack of accuracy, etc. It shall eternally result in the inaccuracy of earth pressure calculation, and then lead to instability of foundation pit.

Exterior Factors that Influenced the Stability of the Supporting. With the gradual improvement of the construction codes and regulations, construction technology and construction quality are continuously improved. But for deep foundation pit supporting, construction technology is still very lacking. The geotechnical materials are different in different regions, so there are many problems in the construction of foundation pit support. For example, the unreasonable dewater and not-timely recharge, etc cause a large number of soil deformation.

The most direct effect is the failure of the supporting system caused by the improper construction, end up as system instability. The site management is not scientific, the support is not timely, the foundation pit is exposed for too long. This eventually led to the variation of strength index of the soil of sidewall, so the foundation pit must be supported during excavation. The inadequate informationization construction and negative monitoring are also important parts of the problem. At the same time, a reliable support type should be chosen in the support scheme, and through scientific and reliable optimization of index to achieve the rationality of selecting. In the past, the instability of many foundation pits was due to the imperfect design of the scheme. And the result has caused serious damage to people and property. Sometimes some accidental factors can result in severe instability of foundation pit, such as earthquake, explosion, strong wind, heavy rain, etc. In short, paying more attention to the changes of external factors and making timely assessment are necessary.

\section{Establishment of Failure Mode}

The research on the stability of deep foundation pit based on the reliability theory must be correct and reasonable. Only the failure mode is correct and the reliability index can get the probability reliability. At present, engineering generally agrees that the failure mode of foundation pit mainly is overturning, bottom heave, overall instability. However, engineering practice has proved that the foundation pit instability mode is far more than these, but a coupling multiple failure mode. The rest of the failure mode is easy to ignore and cause the instability of foundation pit. The paper established a more comprehensive failure mode of foundation pit, which is the foundation for the analysis of the stability of foundation pit.

\section{Because of Soil Mass in the Sidewall of Foundation Pit \\ Bottom Heave of Foundation Pit}

When soil inside the foundation pit constantly being dug out, height difference of soil between the inside and outside of pit soil makes outer soil extrude inwards. It will result in bottom heave of foundation pit soil, then subsidence outside foundation pit. Under the action of gravity and surface overload, the soil on the outside of pit sidewall may crowd to the inner side of the foundation pit from the bottom of the foundation pit. The shear failure of soil will take place, and the shear failure is mainly related to soil shear strength and the loading history. 


\section{Slope Instability of Foundation Pit}

According to the actual situation, some foundation pits are in need of the slope excavation. If the slope design is not reasonable, the foundation pit will also loss stability. The judgement of instability is usually by means of calculating the slope stability. Start with the assumption that sliding circular arc surface, and then calculate the resisting moment and driving moment. If resisting moment is less than driving moment, pit instability will take place.

\section{Insufficient Recognition on Space-Time Effect}

Application of time-space effect theory can effectively control the deformation of foundation pit, and then protect the safety of surrounding buildings, underground pipelines, tunnels and so on. The theory has been widely used in deep foundation pit construction and played an important role [9]. In soft soil area, the effect is very significant. In the deep foundation pit supporting project, the use of time-space effect theory can improve the construction efficiency, save a lot of time and foundation reinforcement cost, obtain significant economic benefits [10].

Deep foundation pit is a three-dimensional space structure, so the research on the stability of foundation pit should belong to the problem of three-dimensional space. Through a large number of engineering practice and numerical simulation analysis, it can be known that the deep foundation pit has obvious space-time effect in the process of excavation. To resolve the problems caused by the space-time effect, foundation pit should be excavated regularly, layer by layer, step by step. Before supporting, the foundation pit can not be exposed for long time, so as to reduce the variation of the soil parameters, and avoid the premature decrease of soil strength.

\section{Because of Supporting Structure}

Different support schemes will adopt different supporting patterns. But no matter what type of supporting structure, they all must meet the requirements of strength and rigidity. And design should base on the deformation control, and this control and monitoring means should be join up to use.

Using bracing structure, the design strength of bracing is not enough. The eccentricity of bracing erection is larger, and can not meet the design requirements. Using tension anchor supporting, the anchoring force is not enough. All of these will lead to the destruction of supporting structure, sometimes with the overall sliding of the foundation pit. If insertion depth of retaining structure was not enough, or the mechanical properties of bottom soil were not good, or the passive earth pressure was small, there will be riser failure for the supporting structure.

The bracing design strength or anchoring force are not enough, or the bracing structure or anchorage system construction is not timely, or external force impact, or the grouting, piling, partial load outside of the foundation pit, etc. All of these will result in toppling of retaining structure toward foundation pit inside.

\section{The Groundwater}

The large proportion of the foundation pit accident is directly or indirectly related to groundwater. The groundwater is the most active element in geological environment, and its influence on the mechanical properties of rock and soil can not be ignored [11].

The dynamic water pressure is relatively weak in the natural state, but due to the change of the natural groundwater dynamic equilibrium conditions in the engineering activities. Under the influence of dynamic water pressure, some geotechnical hazards will often appear. Such as quicksand, piping, uprush, etc.

Under the action of water, the engineering properties of rock and soil mass are often deteriorated. The appearance of this situation may further lead to the failure of the geotechnical engineering projects, then result in more serious consequences. Generally, the rock and soil mass have the characteristics of being soften by water. The shear strength index can be greatly reduced, it will endanger the safety of the project. Meanwhile, the water can also affect the bond force and friction force between the rock and soil mass and the underground structure. 


\section{Correlation Between Failure Modes}

From the point of view of engineering practice and influence factors of deep foundation pit support stability, it is not difficult to find that the most important factor is the groundwater. The groundwater can further affect the mechanical parameters of the soil, and ultimately impact on the strength indexes. The research showed that there is a certain correlation between the strength indexes of soil. To evaluate the stability of supporting structure, it is best to establish the correlation between the failure modes. Through correlation analysis, the main causes of system failure and preventive measures are also obtained. Finally, ensure that the solution of reliability is more close to the engineering practice.

\section{Conclusions}

Through the analysis of the influence factors on the stability of foundation pit, several failure modes of the foundation pit were given. They were divided into three major categories: external rock and soil mass, supporting structure and groundwater. These failure modes are not completely independent. They are coupled with each other and have a certain degree of correlation. Then, the analysis in this paper laid the foundation for the calculation of the foundation pit support system reliability.

\section{References}

[1] Wang Su-guang, The record of accident treatment experience of retaining and protecting for deep foundation pit, first ed., China Machine Press, Beijing, 2005.

[2] Wu Kai, Xu Lv, On deep foundation pit project and analysis of support, Shanxi Architecture. 17 (2011) 67-68.

[3] Jiang Xue-liang, Cao Ping, Application of Analysis on Reliability in Deep Excavation, Journal of Hunan Institute of Science and Technology (Natural Sciences). 3 (2004) 88-91.

[4] Hu Lin, Wang Guo-ti, Wang Hui, Discussion on reliability of retaining structure in deep excavation, Journal of Anhui Technical College of Water Resources and Hydroelectric Power. 2 (2010) 7-8.

[5] Xue Li-ying, Yang Wen-sheng, Li Rong-nian, Discussion and analysis of accident reasons of deep foundation pits, Chinese Journal of Geotechnical Engineering. S1 (2013) 468-473.

[6] Xu Chang-jie, Yin Zhen-yu, The design and case analysis for retaining structure of deep foundation pit, first ed., China Machine Press, Beijing, 2014.

[7] Zhang Zheng, Liu Shu-chun, Zhou Zheng-shen, The variability of rock and soil parameters and its evaluation method, China Civil Engineering Journal. 6 (1995) 43-51.

[8] Huang Guang-long, Wei Min, Li Juan, Analysis of influence of parameters variability on retaining structure stability, Rock and Soil Mechanics. 8 (2010) 2484-2488.

[9] Zhou Guan-nan, Time-space Effect Analysis and Control of Deep Foundation Excavation in Soft Soil, Chinese Journal of Underground Space and Engineering. Z1 (2014) 1653-1658.

[10]Du Hai-bin, Ji Ya-yu, Xiao Shi-lang, Construction Stability Analysis of Super Deep Foundation Pit in Consideration of Time-Space Effect, Building Construction. 8 (2013) 782-784.

[11]Wang Shu-wang, Analysis of the influence of groundwater on the stability of foundation pit, Geotechnical Engineering World. 4 (2006) 52-55. 